\title{
Model and experiments for resonant generation of second harmonic capillary-gravity waves
}

\author{
Adriano Alippi, Andrea Bettucci, Massimo Germano* \\ SBAI Department, Sapienza University of Rome, Via A. Scarpa 16, 00161 Rome, Italy
}

\section{H I G H L I G H T S}

- Experimental proof of 2nd capillarity-gravity harmonic in deep water for 7-60 Hz.

- A new model of local harmonic generation matches results at and out of resonance.

- A threshold effect with respect to water depth has been found.

\section{A R T I C L E I N F O}

\section{Article history:}

Received 23 April 2018

Received in revised form 18 February 2019

Accepted 23 February 2019

Available online 15 March 2019

Communicated by C. Josserand

\section{Keywords:}

Gravity waves

Capillary waves

Resonance condition

Nonlinear acoustics

\begin{abstract}
A B S T R A C T
The generation and propagation of a second harmonic water wave have been investigated in the frequency range between $7 \mathrm{~Hz}$ and $60 \mathrm{~Hz}$ where the velocity vs. frequency curve attains its minimum value. A model is proposed by assuming that the second harmonic is locally generated by point sources on the wavefront of the fundamental wave, and that at any point along the propagation direction the second harmonic be given by the cumulative contribution from all the sources up to the considered point. In the frequency range examined the combined effects of gravity and capillarity yield the so called resonance condition where the fundamental and second harmonic waves share the very same phase velocity. In such a case, wave shape matching condition is maintained between the two waves along all the propagation directions, with the amplitude of the second harmonic only limited by the attenuation effect. Evidence is given experimentally of such effect through the wavenumbers mismatching produced by the model vs. frequency and the detection of the maximum distance of second harmonic amplitude from the wave source. Furthermore, it is found that the resonance condition is a threshold effect with respect to water depth.
\end{abstract}

(c) 2019 Elsevier B.V. All rights reserved.

\section{Introduction}

The nonlinear growth of harmonic components along the propagation direction of an acoustic wave is a long known phenomenon [1], responsible, for instance, of shock wave formation in air [1,2], or in medical lithotripsy applications [3], convolutor signal processing in electronics [4,5], resolution improvement in acoustic microscopy [6-8], as well as in other successfully exploited technological processes [9].

Theoretical approaches to the problem of generating harmonic waves are variously tempted and largely depends on the assumptions that could be correctly made upon the wave nature and the propagation materials. Particularly, inhomogeneous waves present harder solutions, since the sources of nonlinearity are not uniformly distributed over the wavefront planes, even in the case of planar propagation. These are the cases of surface

\footnotetext{
* Corresponding author.

E-mail address: massimo.germano@uniroma1.it (M. Germano).
}

acoustic waves, an important deal in high frequency ultrasonic techniques for electronic applications $[5,10,11]$, or in the much lower frequency range of surface water waves. This last case has been variously studied and approaches have been proposed mainly based on the nonlinear interactions among surface water waves giving rise to an energy transfer among wavetrains affecting wavefield evolution [12-16].

Experiments of previous work are generally relative to the spectra content of the detected signal from the surface elevation in selected points of the field, with no description of the overall surface second harmonic profile. Investigation of the evolution field, indeed, allowed one to set into evidence that the nonlinearity process generates a component of the water elevation that vibrates at double the frequency of the fundamental wave, but does not grow as a true second harmonic wave, since it is constantly generated out of phase with the propagating field, suggesting the effect of local generation. At the resonance conditions alone, the spatial profile follows the one of a true second harmonic, whose growth is only limited by absorption. Experimental 
evidence will be set into evidence, which coherently follows the proposed model.

In the present paper such a model is thus introduced for second harmonic generation of surface water waves, where the generated wave is locally produced proportional to the second power of the fundamental wave and subsequently propagates as an independent wave, producing a wavefront mismatching with the fundamental one, that may be easily detected experimentally. The model is then used to best fit the various physical parameters and a strong evidence is given of the effect produced at the so called resonance condition, where the fundamental wave and its harmonic share the very same phase velocity. Mismatchings between wavenumbers and distance of maximum second harmonic amplitude from the wave source are given vs. wave frequency, which both set into clear evidence the resonance condition.

\section{Second harmonic generation model}

Modeling the generation of a second harmonic wave in case of planar propagation in acoustics can be performed by assuming a distribution of infinitesimal sources to be created along the propagation direction; they are due to any cause of nonlinear behavior of the wave geometry as well as of the medium structure at the fundamental driving frequency, each being proportional to first approximation to the square of the local amplitude and/or stress amplitude and to the infinitesimal volume of interaction and, as such, varying in time as twice the fundamental wave frequency. The model may lead to an analytical result for the second harmonic wave field, as long as its amplitude is limited to values considerably lower than those of the fundamental, so that no feed back of the power should be taken into account that contributes to vary the very same generating terms. The so called triad interaction is then limited to just one way from the most powerful harmonic to the others and, more specifically, to the second harmonic.

Each perturbation produced at any given point will propagate independently from the others as a true second harmonic wave, though limited to an infinitesimal amplitude, with its proper velocity of propagation. At any point, then, along the propagation direction, a second harmonic wave will be present, given by the sum of the contributions from all the sources as generated up to the considered point.

A monochromatic unidirectional plane wave is supposed to propagate along $x$ direction, produced at point $x=0$ with amplitude $A_{1}$ and attenuation coefficient $\alpha$, as

$a_{1}(x, t)=\frac{1}{2} A_{1} e^{-\alpha x}\left[e^{i\left(\omega t-k_{1} x\right)}+e^{-i\left(\omega t-k_{1} x\right)}\right]$.

Within any interval $d x^{\prime}$ positioned around point $x^{\prime}$ a perturbation is produced due to first order nonlinear effect proportional to $a_{1}^{2}$ that propagates along $x$ direction, reaching point $x$ at a time delayed by the lapse it takes a second harmonic wave to travel a space interval equal to $x-x^{\prime}>0$, with its amplitude reduced by the attenuation produced within that very same interval

$$
\begin{aligned}
d a_{2}\left(x, x^{\prime}, t\right)= & \frac{1}{4} \Gamma A_{1}^{2} e^{-2 \alpha x^{\prime}} e^{-\beta\left(x-x^{\prime}\right)} \\
& \times\left\{e^{2 i\left[\omega\left(t-\frac{x-x^{\prime}}{v_{2}}\right)-k_{1} x^{\prime}\right]}\right. \\
& \left.+e^{-2 i\left[\omega\left(t-\frac{x-x^{\prime}}{v_{2}}\right)-k_{1} x^{\prime}\right]}+2\right\} d x^{\prime}
\end{aligned}
$$

$\Gamma$, being a phenomenological complex constant that takes into account the nature of the wave (longitudinal, shear, surface etc.), and that depends upon the propagation medium. It represents how much of the fundamental wave energy, which at any time $t$ is present in the volume interval $d x^{\prime}$, is flowing outward to the second harmonic wave.

Its amplitude describes the efficiency of the nonlinear interaction and its phase relates to the phase lag between the local values of the fundamental and the generated harmonic wave; $\omega$, is the angular frequency of the fundamental component; $k_{1}$ and $k_{2}$, the fundamental and second harmonic wavenumbers, respectively; $v_{2}=2 \omega / k_{2}$, the second harmonic wave velocity of propagation; $\alpha$ and $\beta$, the fundamental and second harmonic component attenuation factors, respectively. A dc term is present within graphs in Eq. (2), resulting from the square of the cos term of the fundamental wave; it locally adds to the second harmonic source term, though it does not propagate. At point $x$ the second harmonic wave takes the form

$$
\begin{aligned}
& a_{2}(x, t)=\int d a_{2}\left(x, x^{\prime}, t\right) \\
& =\frac{\Gamma A_{1}^{2} e^{-\beta x}\left\{e^{2 i \omega\left(t-\frac{x}{v_{2}}\right)}\left[e^{2 i\left(\frac{\omega}{v_{2}}-k_{1}\right) x}-1\right]\right\}}{8 i\left(\frac{\omega}{v_{2}}-k_{1}\right)} \\
& \times \frac{-e^{-2 i \omega\left(t-\frac{x}{v_{2}}\right)}\left[e^{-2 i\left(\frac{\omega}{v_{2}}-k_{1}\right) x}-1\right]}{8 i\left(\frac{\omega}{v_{2}}-k_{1}\right)}
\end{aligned}
$$

that may be finally reduced to

$$
\begin{aligned}
a_{2}(x, t) & =\frac{\Gamma A_{1}^{2} e^{-\beta x}}{2\left(k_{2}-2 k_{1}\right)} \\
& \times \sqrt{\left(\cos 2 k_{1} x-\cos k_{2} x\right)^{2}+\left(\sin k_{2} x-\sin 2 k_{1} x\right)^{2}} \\
& \times \sin \left[2 \omega t+\arctan \left(\frac{\sin k_{2} x-\sin 2 k_{1} x}{\cos 2 k_{1} x-\cos k_{2} x}\right)\right] .
\end{aligned}
$$

The perturbation thus produced oscillates in time with angular frequency $2 \omega$ at any point along the propagation direction; however, it is not to be considered as a true second harmonic wave, since its phase does not vary linearly along the propagation direction as $k_{2} x$, as one would expect, rather through a proper combination of the wavenumbers $k_{1}, k_{2}$. In addition, its amplitude is also modulated by a combination of the wavenumbers $k_{1}, k_{2}$, and is linearly related to the forcing term $\Gamma$ times the square of the fundamental wave amplitude $A_{1}$ and the attenuation factor $e^{-\beta x}$. It is worth noting that the dispersionless case, corresponding to condition $k_{2}=2 k_{1}$, represents the well known resonance effect [17], where there is no modulation of the generated wave, which grows as a true second harmonic wave at a constant rate, as it could be proved by letting $k_{2} \rightarrow 2 k_{1}$, thus giving

$\left|a_{2}(x, t)\right| \cong \frac{1}{2} \Gamma A_{1}^{2} e^{-\beta x} x \cos \left(2 \omega t+k_{2} x\right) \ll A_{1}$.

It is useful to recall that it has been shown that resonance conditions are not possible in case of deep water gravity waves [16], and that the condition can be verified [17-19] only if capillarity terms are introduced. Capillarity waves, indeed, are of interest in remote sensing techniques, and in the generation of water waves by wind, having the highest efficiency [20].

This case clearly sets the limit for the model to satisfy the above mentioned condition of validity, where the generated harmonic wave maintains its amplitude well below that of the generating fundamental. That is up to the point $x$ satisfying the condition

$x e^{-\beta x} \ll \frac{2}{\Gamma A_{1}}$. 


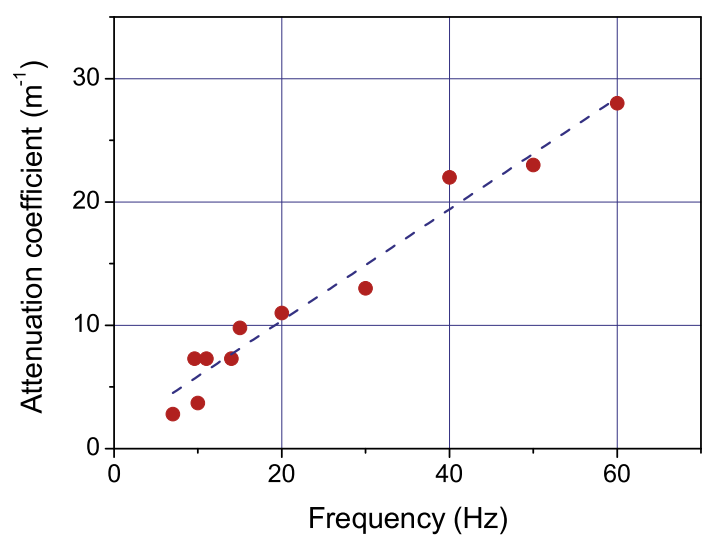

Fig. 1. (Color online) Attenuation coefficient $\alpha$ vs. driving frequencies used in the experiment; Linear fit of the data is displayed as a dashed line.
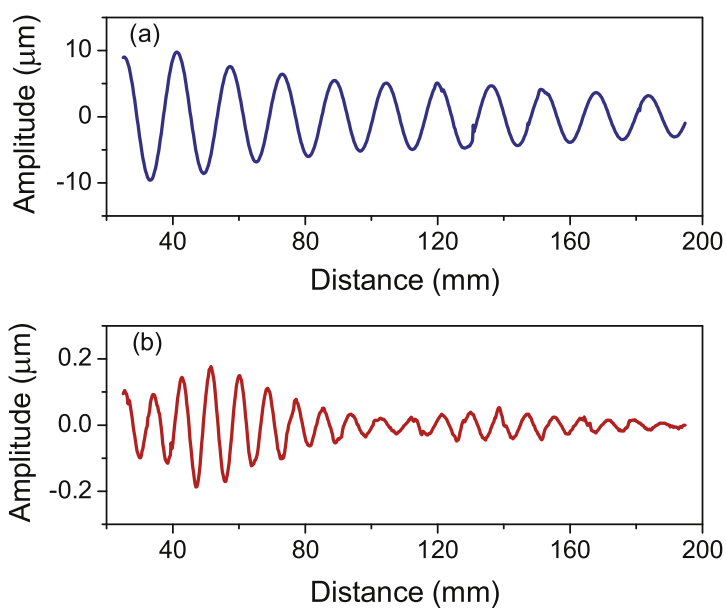

Fig. 2. (Color online) Waveforms of the fundamental (a) and second harmonic wave (b), vs. distance $x$ from the piston-like source oscillating at frequency $f_{1}=14 \mathrm{~Hz}$.

\section{Experimental results and discussion}

Surface waves are produced on deionized water by horizontally moving as a harmonic motion a thin aluminum plate (source transducer), $15 \mathrm{~cm}$ wide and $2 \mathrm{~mm}$ thick, vertically set into the water and partially immersed by an amount $d=4 \mathrm{~mm}$ into the liquid [21]. Oscillatory frequencies produced by a piston like source, properly connected to the plate, can be varied from dc to few tens of hertz in a linear regime, with amplitudes sufficiently high to produce harmonic, as well as subharmonic waves.

Previous to experiments on the nonlinear generation efficiency of the water waves, spurious nonlinear generation directly produced at the plate source was carefully tested and no output was detected relative to such effect, though the meniscus oscillatory deformation was found to be responsible for the generation efficiency of the fundamental mode [22]. This effect, however, has no influence on the efficiency of the second harmonic generation, once the fundamental wave has been produced. In addition, water surface contamination has been carefully taken into account, and a measurement of the absorption coefficient $\alpha$ in the frequency range $7-60 \mathrm{~Hz}$ has been carried out (see Fig. 1). Values fully match the literature parameters [23], making us confident of the validity of the experimental conditions.

The oscillatory motion of the water surface is detected with a laser optical probe and the scanning of the surface by means of a point-to-point motor along the axis of the source transducer.
The signal is then properly treated to perform complex Fourier analysis of each single component. Experiments are all performed in a water tank $40 \times 40 \mathrm{~cm}$ by size, with water depth equal to $h=10 \mathrm{~mm}$. Typical results of a line scanning of the water surface along the transducer axis are given in Fig. 2 for the fundamental, (a), and its second harmonic, (b), wave component at excitation frequency $f_{1}=14 \mathrm{~Hz}$. The fundamental wave profile evidently proves the attenuation of the wave while the second harmonic feels the modulation due to the phase lag at each $x$ position described above between the generated wave and the locally generated one. A best fit is subsequently produced between the waveforms from the experiment and the model.

It should be pointed here that the dispersive profile would not be the same, would have the two waves being produced independently one from the other. That also justifies the application of the model even to the generation of harmonic waves at frequencies different from that of the so-called resonance, in as much as there is no triadic interaction of the waves, but a local generation that matches always and at any point the fundamental. This is performed by successively varying the dispersion parameter $\Delta k=k_{2}-2 k_{1}$, the absorption $\beta$ (supposedly equal to $2 \alpha$ ) and the coupling coefficient $\Gamma$. It should be mentioned that the first two are quite independent one from the other, since dispersion is responsible for the modulation periodicity, while absorption modulates the overall shape of the waveform. They are then easily best fitted from the waveform shape. The coupling coefficient $\Gamma$, in addition, increases the maximum attained value of the waveform as far as its amplitude value is concerned, while its phase is responsible for the phase matching between the fundamental and the second harmonic waveforms.

As an example, Fig. 3 reports the best fitting result of the second harmonic wave shape along propagation direction (Fig. 3a) and, for evidence sake of the sensitivity to the various parameters, the same fitting performed when changing the phase $\gamma$ alone of the coupling factor $\Gamma$ from the best fitting case $(\gamma=2.8$ in Fig. 3a) to a different value ( $\gamma=2.0$ in Fig. 3b). A substantial difference is evident in the phase matching, that makes us confident of the robustness of the method. A series of measurements and the relative best fitting curves have been done for water wave frequencies within the range $7 \mathrm{~Hz}<f<60 \mathrm{~Hz}$. The dispersion curve of water waves is theoretically evaluated through the use of the expression

$\omega^{2}=\left(g k+\frac{\tau}{\rho} k^{3}\right) \tanh k h$

and it is plotted as the velocity term $v=\omega / k$ versus frequency in Fig. 4 for the value of the water surface tension $\tau$ equal to $72 \times 10^{-3} \mathrm{~N} \mathrm{~m}^{-1} ; \mathrm{g}$ is the gravity acceleration and $\rho$ the water density. With regard to harmonic wave generation, it is worth to note that at the frequency $f_{\text {res }}=9.8 \mathrm{~Hz}$ the wave shares the same phase velocity with its second harmonic $f_{2}=$ $19.6 \mathrm{~Hz}$ (see Fig. 4). This is the situation generally recalled as the resonance condition [24], where matching of the wave shape is maintained throughout all the propagating distance, producing the highest value of the second harmonic, only limited by attenuation effects $[16,17]$. Our experimental conditions produce a well defined one-dimensional wave propagation without twodimensional evolution of the wave front such as in Henderson \& Hammack [14]. Taking the product between the wavemaker amplitude stroke $(s)$ and the wavevector of the fundamental $\left(k_{1}\right)$ as a parameter measuring of the nonlinearity in the generation process, our experimental conditions are in the range $0.02 \leq$ $s k_{1} \leq 0.15$ that is well below the values $\left(s k_{1}>0.20\right)$ for the appearance of a wide variety of lengthscales in the water surface pattern [14]. The absence of other frequencies except $f_{1}$ and $2 f_{1}$ is shown in Fig. 5 that reports the frequency content of the wave 

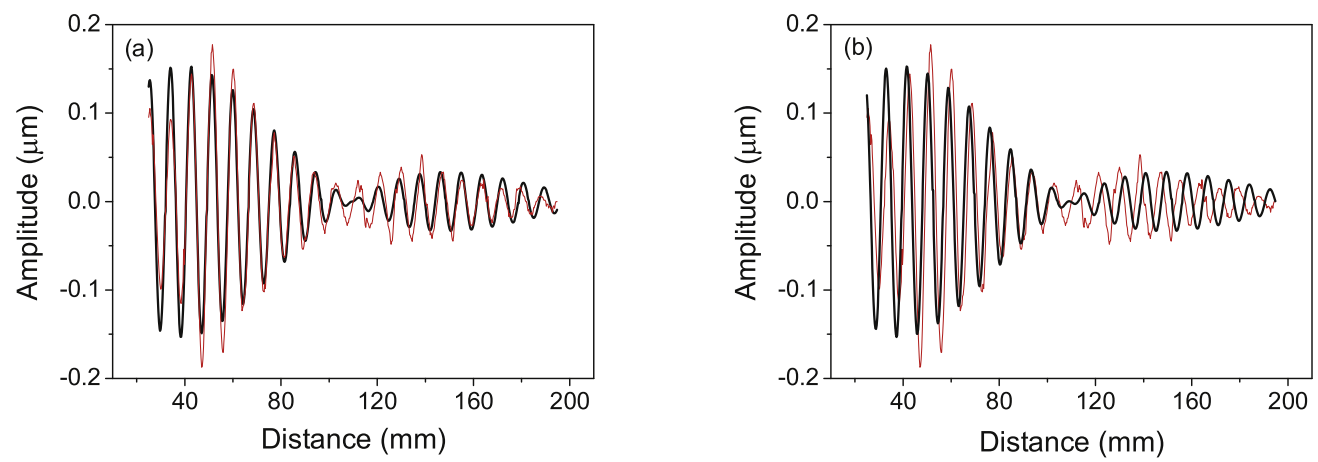

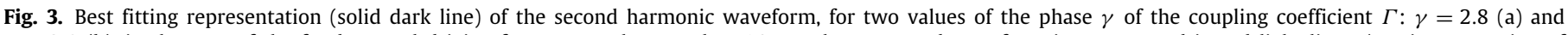

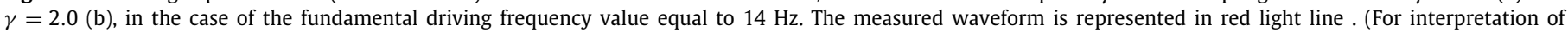
the references to color in this figure legend, the reader is referred to the web version of this article.)

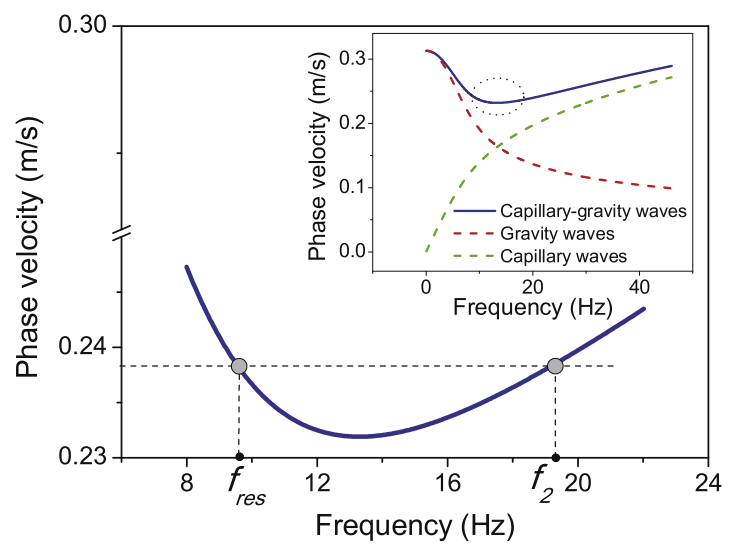

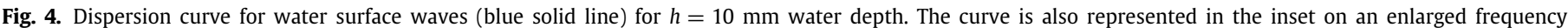

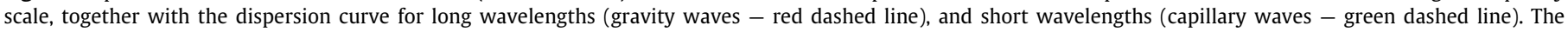

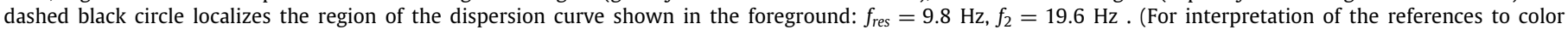
in this figure legend, the reader is referred to the web version of this article.)

elevation taken along the transducer axis and off-axis for different excitation frequencies $f_{1}$.

Evidence of the resonance effect is given in Fig. 6, where the mismatching effect is reported as the ratio $n=k_{2} /\left(k_{2}-2 k_{1}\right)$ between second harmonic wavenumber and the wavenumber mismatching; experimental values are reported (closed circle) together with those from the fitting model (open circle). Continuous line represents the function $v\left(f_{1}\right) /\left[v\left(f_{1}\right)-v\left(f_{2}\right)\right]$, as it can be obtained from the analytical expression given by Eq. (6). The overall correct fitting between the series of data enforces the confidence in the model adopted, that may be well set into use for further development of nonlinear phenomenology of water surface waves. It has to be noted that resonance in dispersive wave propagation can only be attained in case that the velocity curve vs. frequency presents a stationary point with zero derivative and that is possible in surface water waves because of the converging effect of capillarity and gravity. For sake of evidence the inset in Fig. 4 reports the wave velocity curve in the hypothetical case that only one of the effects be present separately, corresponding to the extreme cases of low and high frequency waves.

Monotonic dependence of dispersion curve vs. frequency cannot produce resonance conditions, as they are truly possible in our case. Additional evidence of the resonance condition is given in Fig. 7, where the distance from the oscillating plate where the maximum second harmonic wave amplitude is attained is reported vs. second harmonic wave frequency. At the resonance condition frequency $\left(f_{2}=19.6 \mathrm{~Hz}\right)$, the growth of the second harmonic wave is only limited by attenuation effects and not by dispersion, thus allowing the amplitude to grow farther away with respect to other frequencies. It is worth stating that resonance condition is a threshold effect with respect to the water depth $h_{t h}$, once that a particular liquid (water, in our case) has been selected. It is given by the relation:

$h_{t h}=\sqrt{\frac{3 \tau}{\rho g}}$

and is easily obtained by setting equal to zero the second derivative of the velocity curve with respect to the wavenumber. The value for water waves is $h_{\text {th }} \simeq 4.7 \mathrm{~mm}$ and slightly changes with the surface tension parameter $\tau$. Once the resonance is available, the fundamental and second harmonic wave frequencies localize at the left and the right position with respect to the curve velocity minimum, represented in Fig. 4. Fig. 8 reports the value of the frequency for which such a minimum is attained versus water depth: it can be seen that no minimum is attained below the threshold value $h_{\text {th }} \simeq 4.7 \mathrm{~mm}$ and that there is an asymptotic value of the frequency $\left(f_{\text {res }}=9.6 \mathrm{~Hz}\right)$ at increasing depths. It is worth to focus that the value of $h_{t h}$ is equal to the water depth for which water waves behave almost as dispersionless waves for a great range of wavelengths [25]. Actually only for water depths higher than $h_{t h}$ the dispersion curve shows a finite minimum value, so as to satisfy resonance condition. This has been found experimentally, by decreasing the water depth below $5 \mathrm{~cm}$.

It might be of interest to note that the threshold depends upon the inertial forces, as well, as it is suggested by the $g$ factor in Eq. (7). That means that in case of not inertial systems or, more practically, in case of rotating liquids, resonance conditions may 

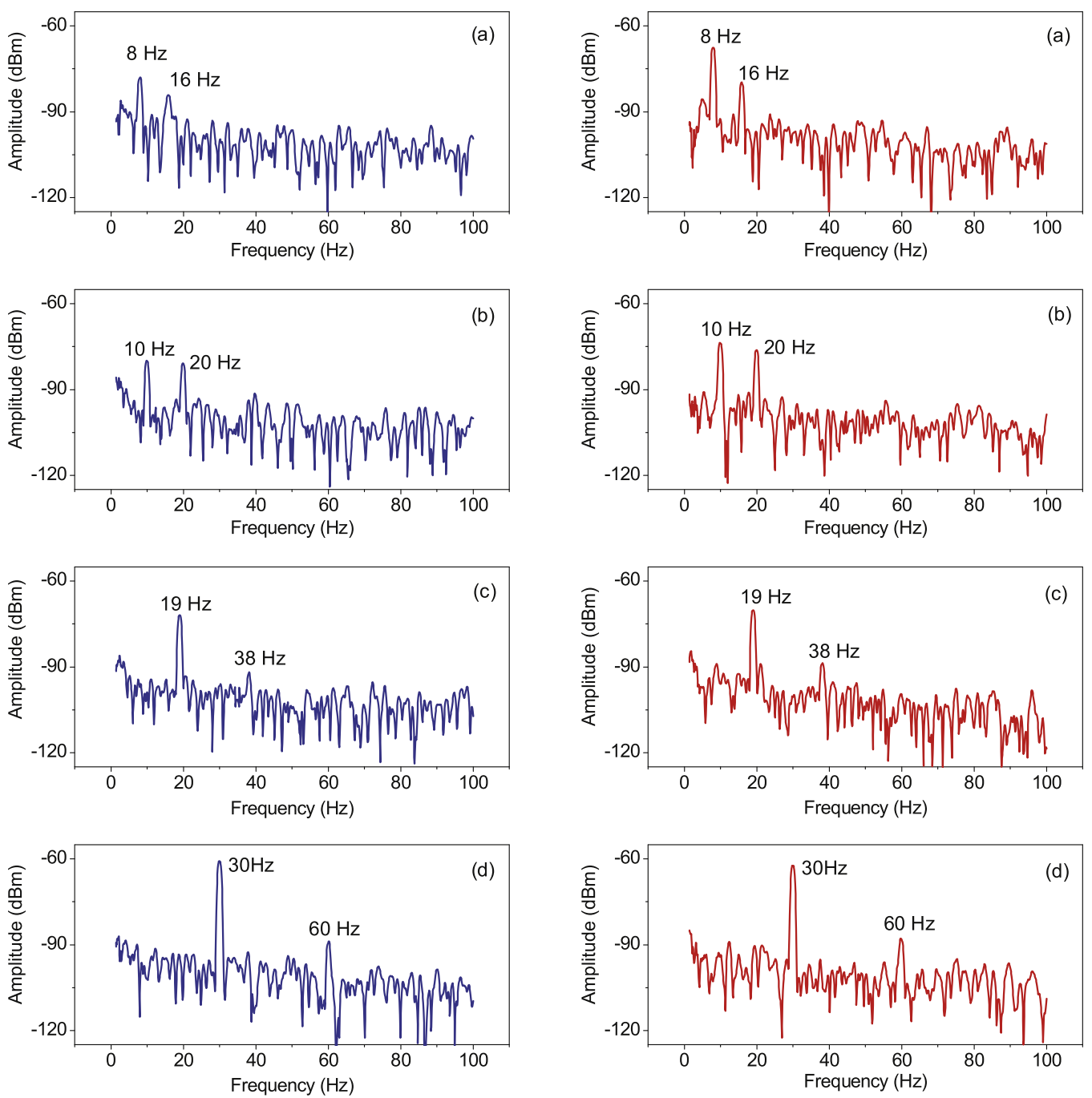

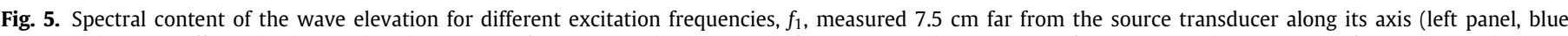

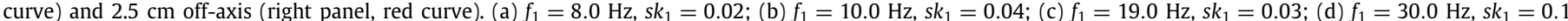
. (For interpretation of the references to color in this figure legend, the reader is referred to the web version of this article.)

be canceled or, the other way around, enforced. That may particularly affect subharmonic generation, and chaos development there hence, where reinforcement of the effect is more easily achievable in resonance conditions.

\section{Conclusion}

In the present paper evidence has been given of the resonant generation of second harmonic water waves in the frequency range between $7 \mathrm{~Hz}$ and $60 \mathrm{~Hz}$. At the resonance condition, the combined effects of gravity and capillarity - which produces a minimum in the dispersion curve of water waves - cause the fundamental and second harmonic waves to share the very same phase velocity. In such a case, the wave shape matching condition is maintained between the two waves along the propagation direction, producing the highest value of the second harmonic wave amplitude, only limited by attenuation effects. A theoretical model is presented in which the second harmonic wave has been assumed to be locally generated, due to the nonlinear behavior of the propagating medium, by point sources on the wavefront of the fundamental wave. At any given point along the propagation direction, the amplitude of the second harmonic wave has been supposed to be produced by the cumulative contribution from the second harmonic water waves produced by point sources

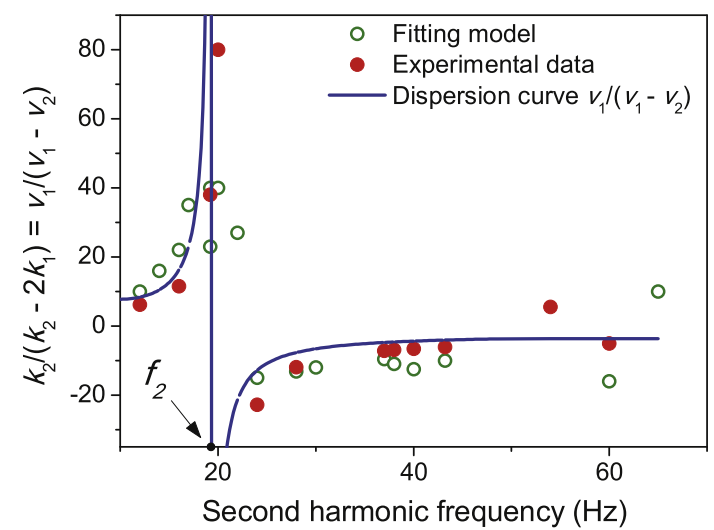

Fig. 6. (Color online) Parameter $n=k_{2} /\left(k_{2}-2 k_{1}\right)=v_{1} /\left(v_{1}-v_{2}\right)$ of the mismatching condition between second harmonic and fundamental wave. Resonance condition $\left(k_{2} \simeq 2 k_{1}\right)$ is attained at frequency $f_{2}=2 f_{\text {res }}=19.6 \mathrm{~Hz}$. Open circles refer to the fitting model, closed circles to the experimental values and solid line to the dispersion curve.

up to the considered point. The model provides that resonance condition is a threshold effect with respect to water depth. 


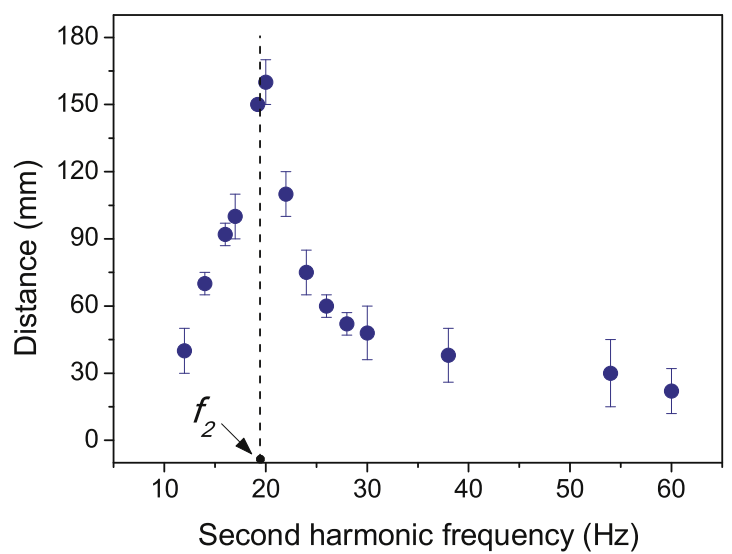

Fig. 7. (Color online) Distance $x$ from the piston-like source where the maximum value of the amplitude of the second harmonic surface water wave is attained. At the resonance condition $\left(f_{2}=2 f_{\text {res }}=19.6 \mathrm{~Hz}\right)$ second harmonic wave amplitude is only limited by the attenuation factor, attaining its maximum value farther away from the source compared to off resonance conditions, where second harmonic wave amplitude is modulated by a combination of $k_{1}$ and $k_{2}$ wavenumbers.

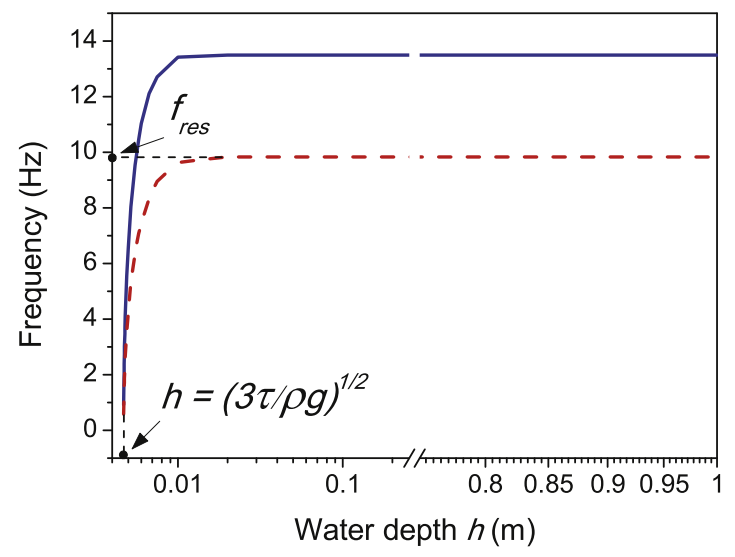

Fig. 8. (Color online) Frequency value of the minimum water surface wave velocity (blue line, continuous) and of the fundamental resonant wave (red line, dashed) vs. water tank depth, with threshold value at about $4.7 \mathrm{~mm}$.

Second harmonic resonant generation around $9.8 \mathrm{~Hz}$ has been put in evidence through the mismatching condition between the wavenumber of the fundamental and second harmonic waves vs. frequency, as calculated from the model, and the detection of the maximum distance from the wave source where the second harmonic attains its maximum value.

\section{References}

[1] M. Hamilton, D.T. Blackstock, Nonlinear Acoustics, Academic Press, San Diego, CA, 1998.

[2] G. Witham, Linear and Nonlinear Waves, John Wiley \& Sons, 1974.

[3] S.N. Gurbaton, O. Rudenko, A. Saichev, Waves and Structures in Nonlinear Nondispersive Media General Theory and Applications to Nonlinear Acoustics, Springer Berlin Heidelberg, 2012.

[4] S.K. Mitra, G. Sicuranza, Nonlinear Image Processing, Academic Press, 2001.

[5] G. Kino, Acoustic Waves: Devices, Imaging, and Analog Signal Processing, Prentice-Hall, Englewood Cliffs, NJ, 1987.

[6] A. Briggs, Acoustic Microscopy, Oxford University Press, Oxford New York, 2010.

[7] R.G. Maev, Acoustic Microscopy: Fundamentals and Applications, WileyVCH, 2008.

[8] D. Rugar, Resolution beyond the diffraction limit in the acoustic microscope: A nonlinear effect, J. Appl. Phys. 56 (5) (1984) 1338-1346, http: //dx.doi.org/10.1063/1.334124.

[9] T. Kundu, Ultrasonic Nondestructive Evaluation: Engineering and Biological Material Characterization, CRC Press, 2004.

[10] R. Weigel, D. Morgan, J. Owens, A. Ballato, K. Lakin, K. Hashimoto, C Ruppel, Microwave acoustic materials, devices, and applications, IEEE Trans. Microw. Theory 50 (3) (2002) 738.

[11] A. Alippi, A. Palma, L. Palmieri, G. Socino, Determination of coupling coefficient in second harmonic generation of acoustic surface waves, J. Appl. Phys. 45 (10) (1974) 4347-4349, http://dx.doi.org/10.1063/1.1663055.

[12] J.R. Wilton, On ripples, Philos. Mag. Ser. 629 (1915) 688-700.

[13] L.F. McGoldrick, An experiment on second-order capillary gravity resonant wave interactions, J. Fluid Mech. 40 (1970) 251-271.

[14] D. Henderson, J.L. Hammack, Experiments on ripple instabilities part 1. resonant triads, J. Fluid Mech. 184 (1987) 15-41.

[15] L. Debnath, Nonlinear Water Wave, Academic Press, 1994.

[16] O.M. Phillips, On the dynamics of unsteady gravity waves of finite amplitude part 1. the elementary interactions, J. Fluid Mech. 9 (02) (1960) 193, http://dx.doi.org/10.1017/s0022112060001043.

[17] J.L. Hammack, D.M. Henderson, Resonant interactions among surface water waves, Annu. Rev. Fluid Mech. 25 (1) (1993) 55-97, http://dx.doi.org/10. 1146/annurev.fl.25.010193.000415.

[18] L.F. McGoldrick, Resonant interactions among capillary-gravity waves, J. Fluid Mech. 21 (02) (1965) 305, http://dx.doi.org/10.1017/ s0022112065000198.

[19] F. Dias, C. Kharif, Nonlinear gravity and capillary-gravity waves, Annu. Rev. Fluid Mech. 31 (1) (1999) 301-346, http://dx.doi.org/10.1146/annurev.fluid. 31.1.301.

[20] A. Ramamonjiarisoa, S. Baldy, I. Choi, Laboratory Studies on Wind-Wave Generation, Amplification and Evolution, Vol. IIa, Springer Science + Business Media, New York, 1978, pp. 403-420.

[21] A. Bettucci, M. Germano, A. Alippi, Resonant condition in second harmonic generation of water surface waves, in: P. Blan-Benon, V.W. Sparrow, D. Dragna (Eds.), Recent Developments in Nonlinear Acoustics, AIP Publishing, Melville, 2015, p. 090006

[22] C.Y. Lee, J.A. Tallmadge, Meniscus shapes in withdrawal of flat sheets from liquid baths. dynamic profile data at low capillary numbers, Ind. Eng. Chem. Fundam. 13 (4) (1974) 356-360, http://dx.doi.org/10.1021/ i160052a011.

[23] T. Eadkhong, S. Danworaphong, Imaging surface water waves for the determination of surface tension and spatial attenuation, Can. J. Phys. 92 (2014) 141-144.

[24] W.F. Simmons, A variational method for weak resonant wave interactions, Proc. R. Soc. Lond. Ser. A 309 (1499) (1969) 551, http://dx.doi.org/10.1098/ rspa.1969.0056.

[25] M.J. Lighthill, Waves in Fluids, Cambridge University Press, Cambridge, UK New York, 2001. 